along both pathways and 1 deemed clinically not suitable for TAVI after initial investigation. Of the 44 outpatients, 28 patients were managed in pathway 1 (1.8 patients per month) and 16 in pathway 2 (2.3 patients per month). The mean time from entry to pathway to procedure was 221 days in pathway 1 and 87 days in pathway $2(\mathrm{p}<0.001)$. $94 \%$ of outpatients in pathway 2 were treated within the 18 -week target compared to only $19 \%$ in pathway 1 . There were 5 adverse events in pathway 1 but no adverse events in pathway 2 (figure 1 ).

Conclusions Improving our local TAVI pathway has led to a significant reduction in the time to treatment. Most outpatients now undergo TAVI well within the BCIS 18-week target and 68 days faster than the UK average. Though the numbers are small, we also demonstrated an associated reduction in adverse events. These findings suggest that adopting a dedicated structural clinic model has led to significant improvements at DGH level, ultimately resulting in reducing the time to TAVI and improving patient care.

Conflict of Interest None

\section{BIVENTRICULAR ASSESSMENT AND QUANTIFICATION OF PRIMARY MITRAL REGURGITATION IS FEASIBLE AND REPRODUCIBLE DURING CONTINOUS SUPINE EXERCISE CARDIOVASCULAR MAGNETIC RESONANCE}

${ }^{1}$ Thomas Craven, ${ }^{1}$ Nicholas Jex, ${ }^{1}$ Miroslawa Gorecka, ${ }^{2}$ David Higgins, 'Louise Brown, ${ }^{1}$ Arka Das, ${ }^{1}$ Amrit Chowdhary, ${ }^{1}$ Sharmaine Thirunavukarasu, ${ }^{1}$ Noor Sharrack, 'Erica Dall'Armellina, ${ }^{3}$ Eylem Levelt, ${ }^{1}$ Peter Swoboda, 'Sven Plein, 'John Greenwood. ${ }^{1}$ University of Leeds, Leeds, UK; ${ }^{2}$ Philips; ${ }^{3}$ University of Leeds, Multidisciplinary Cardiovascular Research Centre and Biomedical Imaging Science

\subsection{6/heartjnl-2021-BCS.15}

Background Biventricular volume and great vessel flow assessment during continuous supine free-breathing exercise cardiovascular magnetic resonance (Ex-CMR) has recently been validated in healthy volunteers using Compressed SENSEx3 (CS3) sequences. Exercise transthoracic echocardiography (TTE) provides prognostic information in primary mitral regurgitation (MR). Resting CMR offers reference standard biventricular assessment and MR quantification with superior reproducibility to TTE. Ex-CMR assessment of biventricular volumes and quantitated MR may offer additional prognostic information in primary MR. Therefore we aimed to determine the feasibility of biventricular assessment and MR quantification in primary MR patients during continuous supine ExCMR using CS3 sequences.

Methods 10 asymptomatic patients with at least moderate primary MR on TTE ( 8 male, median age 62, 55-67years interquartile range) underwent continuous in-scanner (1.5T Philips Ingenia) supine cycle ergometer (Lode BV) Ex-CMR. Target heart rates (THR) were individually prescribed using heart rate reserve (HRR) and age predicted maximal heart rate model. Participants exercised for 2-minutes at no resistance, then an increase of 25-Watts every 2-minutes until THR achieved at low (30-39\% HRR), then moderate (40-59\% HRR) exercise. CMR imaging: free-breathing CS3 respiratory navigated short axis cine imaging and free-breathing CS3 aortic phase-contrast magnetic-resonance at rest, low and moderate exercise stages. MR was quantified indirectly from LV and aortic stroke volumes. Intra/inter-observer reproducibility was assessed by coefficient of variance $(\mathrm{CV})$.
Abstract 15 Table 1 Haemodynamicand cardiac indices at rest and during supine Ex-CMR

\begin{tabular}{lllll}
\hline & \multicolumn{2}{l}{ Exercise Stage } & p-value \\
\hline & Rest & Low & Moderate & \\
\hline Heart rate (bpm) & $61 \pm 10$ & $98 \pm 6$ & $115 \pm 6$ & $<0.001$ \\
LV-end-diastolic volume $\left(\mathrm{ml} / \mathrm{m}^{2}\right)$ & $112 \pm 23$ & $111 \pm 21$ & $107 \pm 22$ & 0.185 \\
LV-end-systolic volume $\left(\mathrm{ml} / \mathrm{m}^{2}\right)$ & $41 \pm 12$ & $36 \pm 9.8$ & $37 \pm 11$ & 0.055 \\
LV ejection fraction $(\%)$ & $64 \pm 4.9$ & $67 \pm 5.1$ & $66 \pm 6.1$ & 0.075 \\
MR-volume (ml) & $56 \pm 25$ & $52 \pm 23$ & $42 \pm 24$ & 0.032 \\
MR-fraction (\%) & $40 \pm 14$ & $36 \pm 11$ & $30 \pm 15$ & 0.006 \\
RV-end-diastolic volume $\left(\mathrm{ml} / \mathrm{m}^{2}\right)$ & $86 \pm 16$ & $87 \pm 15$ & $87 \pm 12$ & 1 \\
RV-end-systolic volume $\left(\mathrm{ml} / \mathrm{m}^{2}\right)$ & $38 \pm 8.0$ & $34 \pm 5.0$ & $32 \pm 6.5$ & 0.122 \\
RV-ejection fraction $(\%)$ & $55 \pm 5.4$ & $60 \pm 6.0$ & $63 \pm 6.6$ & 0.001 \\
\hline
\end{tabular}

Abbreviations: Ex-CMR, Exercise Cardiovascular magnetic resonance; HR, heart rate; $i$ Indexed to body surface area; LV, left ventricle; MR, mitral regurgitant; RV, right ventricle.

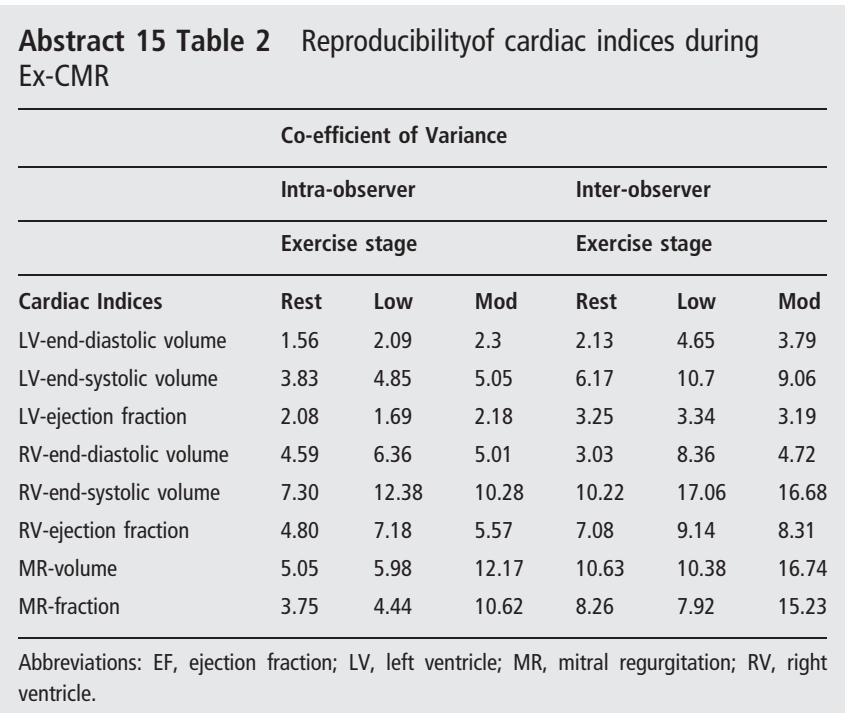

Results All patients completed the Ex-CMR protocol without complication. During exercise, there were no statistically significant changes in biventricular volumes or global left ventricular ejection fraction (LVEF) (table 1). From rest to low and moderate exercise: right ventricular ejection fraction increased $(55 \pm 5.4 \%$ to $60 \pm 6.0 \%$ and $63 \pm 6.6 \%$ respectively, $\mathrm{p}=0.001)$ and MR fraction decreased $(40 \pm 14 \%$ to $36 \pm 11 \%$ and 30 $\pm 15 \%$ respectively, $p=0.006$ ). Intra-observer reproducibility (table 2) was excellent (CV <10\%) except MR fraction \& volumes during moderate and right ventricular end-systolic volumes (RVESV) during both exercise stages, which were good (CV10-20\%). Inter-observer reproducibility (table 2) was excellent $(\mathrm{CV}<10 \%)$, except RVESV and MR volumes at all stages, left ventricular end-systolic volumes during low and MR fraction during moderate exercise, which were good (CV 10-20\%).

Conclusion Biventricular assessment and MR quantification during continuous supine Ex-CMR is feasible and reproducible in asymptomatic primary MR patients. Research assessing the techniques prognostic ability in primary MR patients is now warranted.

Conflict of Interest Nil 